Ann. Zootech., I969, 18 (3), 263-276.

\title{
L'ANÉMIE ET LA COULEUR DE LA VIANDE CHEZ LES VEAUX A L'ENGRAIS DE LA RACE PIE ROUGE DE LA FLANDRE ORIENTALE (')
}

\author{
W. EECKHOUT, M. CASTEELS $\left({ }^{2}\right)$ et F. BUYSSE \\ avec la collaboration technique de Annie Lateur et Anny Van Hecke \\ Station de Recherches de l'État pour l'Alimentation du Bétail, \\ Gontrode (Belgique)
}

SOMMAIRE

La teneur en hémoglobine $(n=257)$ et la valeur de l'hématocrite $(n=264)$ ont été étudiées chez des veaux mâles âgés d'environ ro jours, de la race Pie rouge de la Flandre orientale. Il est apparu que ces deux caractéristiques du sang peuvent différer très fortement chez les veaux de cet âge. Il a aussi été démontré qu'un certain pourcentage de veaux présente dès cet âge un état d'anémie prononcée. La teneur en hémoglobine et la valeur de l'hématocrite baissent pendant la période où les veaux sont engraissés à l'aide d'un aliment d'allaitement contenant environ 20 p.p.m. de $\mathrm{Fe}$ et dissous dans une eau à o,65 p.p.m. de fer.

Cette baisse est cependant très prononcée chez des animaux présentant de fortes teneurs en hémoglobine et des valeurs de l'hématocrite élevées au moment où ils entrent dans la période d'engraissement. On ne relève qu'une faible baisse de la teneur en hémoglobine et de la valeur de l'hématocrite chez les veaux déjà nettement anémiques au début de cette période. L'influence de diverses teneurs en fer de l'aliment d'allaitement sur la teneur en pigment du muscle a été étudiée. Il est apparu que, surtout quand le lait reconstitué n'a qu'une faible tcneur en fer, l'influence du fer sur la couleur de la viande ne se manifeste pas toujours quand il n'est pas tenu compte de la teneur en hémoglobine ou de la valeur de l'hématocrite du sang au début de la période d'engraissement.

Cette influence est cependant très grande car, pour la plupart des teneurs en fer de l'aliment d'allaitement que l'on a testées (à l'exception des très fortes teneurs en fer, c'est-à-dire de 75 à 85 p.p.m.), la viande était nettement plus riche en pigment chez les animaux qui présentaient dès le début des taux d'hémoglobine et des valeurs de l'hématocrite supérieurs à la moyenne, que chez les veaux entrés dans la période d'engraissement avec des taux d'hémoglobine et des valeurs de l'hématocrite inférieurs à la moyenne. Ceci signifie donc que la condition initiale du sang détermine, du moins en partie, la couleur finale de la viande.

(1) Communication no I78 de la Station de Recherches de l'État pour l'Alimentation du Bétail.

(2) Groupe de travail : Amélioration de la qualité de la viande bovine (Président : Pr. Dr. J. Martin). 


\section{INTRODUCTION}

Des expériences précédentes ont montré que la teneur en hémoglobine et la valeur de 1'hématocrite du sang baissent pendant la période d'engraissement ( J. CHARPentier, ig66 ; Schmitten et NoAck, I967 ; Verbeke et Martis, i 967 ; Iieckhout et coll., I969).

Certains auteurs (Roy et coll., I964 ; BI.AXTER et coll., I957) ont montré d'autre part que des veaux exclusivement nourris au lait entier et chez lesquels la consommation de Fe provenant dı milieu est négligeable, peuvent présenter à la longue de très faibles taux d'hémoglobine et des valeurs de l'hématocrite très basses. Dans de telles conditions, la croissance de ces animaux gravement anémiques était nettement inférieure à celle des animaux qui recevaient dı fer, en plus du lait entier. La formule sanguine de ces animaux traités aux préparations ferreuses était aussi favorable, c'est-à-dire que leurs taux d'hémoglobine et leurs valeurs de l'hématocrite étaient plus élevées.

Charpentier et Bonhomme (I968) ainsi que VAx dE VOORDE (I968) ont également montré que la croissance de veaux anémiques recevant des aliments d'allaitement est en général moins favorable que celle de veaux non anémiques. CHARPENTIER et BonHomme ( 9668 ) ont mis par ailleurs en évidence que les veaux très anémiés sont en général abattus à un poids relativement faible.

La première partie de ce rapport est consacrée à l'étude de la teneur en hémoglobine et de la valeur de l'hématocrite du sang de veaux âgés d'environ Io jours, de la race Pie rouge de la Flandre orientale, examinés au début de la période d'engraissement. Nous avons aussi examiné dans quelle mesure la teneur en hémoglobine et la valeur de l'hématocrite diminuaient pendant la période d'engraissement à un aliment d'allaitement pauvre en fer, selon que les teneurs en hémoglobine et les valeurs de l'hématocrite relevées au début de la période d'engraissement étaient élevées ou basses.

La deuxième partie de ce travail traite du problème de la couleur de la viande des veaux à l'engrais en fonction de la teneur en fer de l'aliment d'allaitement ainsi que de la teneur en hémoglobine et de la valeur de l'hématocrite du sang au début de la période d'engraissement.

\section{MÉTHODES}

La teneur en hémoglobine et la valeur de l'hématocrite ont été déterminées sur des échantillons de sang prélevés dans la veine jugulaire et recueillis dans des tubes héparinés.

L'hémoglobine a été déterminée le même jour par la méthode de l'hématine alcaline (Gorther et De GraAfF, 1955).

La valeur de l'hématocrite a été relevée après 30 minutes de centrifugation à 3500 tours par minute dans des tubes de Wintrobe. La teneur en pigment musculaire a été déterminée par la méthode de HorNSEY (I956), sur des échantillons de Longissimus dorsi prélevés sur la carcasse à la hauteur de la $6^{\mathrm{e}}$ côte le jour suivant l'abattage. La myoglobine et l'hémoglobine résiduelle sont déterminées ensemble de cette façon et les résultats sont exprimés en p.p.m. d'hématine.

Les déterminations du fer présent dans l'aliment d'allaitement et dans l'eau ont été effectuées, après minéralisation humide selon PyCK et coll. (1958), par la méthode à l'orthophénanthroline de Bandemer et Schaible ( (944). Pour la détermination du fer de l'eau, roo ml ont d'abord été desséchés par évaporation et le résidu sec a ensuite subi la minéralisation humide, comme cidessus. 


\section{RÉSULTATS}

1. - Les caractéristiques sanguines de veaux mâles de la race Pie rouge de la Flandre orientale au début de la période d'engraissement (environ Io jours)

\section{Hémoglobine.}

Nous disposions pour nos recherches sur la teneur en hémoglobine des veaux de la race Pie rouge de la Flandre orientale, âgés d'environ ro jours, de 257 données en provenance d'un nombre identique d'animaux achetés dans le commerce à un poids d'environ $48 \mathrm{~kg}$. L,es résultats de ces recherches sont exposés dans le tableau I et dans la figure $\mathrm{r}$. Ces résultats nous montrent que la teneur en hémoglobine du sang de très jeunes veaux peut varier dans des limites très larges. Nous avons relevé pour nos animaux une moyenne de I2, I5 g d'hémoglobine par Ioo $\mathrm{ml}$ de sang.

\section{TABLEAU I}

Tencurs on hémoglobine du sang $(\mathrm{g} / \mathrm{r}$ oo $\mathrm{ml})$ de veaux de la race Pie rouge de la Flandre orientale âgés d'environ 10 jours $(n=257)$

\begin{tabular}{|c|c|c|c|}
\hline $\begin{array}{c}\text { Classes hémoglobine } \\
(g / 100 \mathrm{mll})\end{array}$ & $\begin{array}{c}\text { Nombre } \\
\text { d'animaux }\end{array}$ & $\begin{array}{l}\text { Nombre } \\
\text { en p. } 100\end{array}$ & \\
\hline $5,00-5,99$ & 1 & 0,39 & \multirow{5}{*}{13,23} \\
\hline $6,00-6,99$ & 2 & 0,78 & \\
\hline $7,00-7,99$ & $: 3$ & 1,27 & \\
\hline $8,00-8,99$ & 10 & 3,89 & \\
\hline $9,00-9,99$ & 18 & 7,00 & \\
\hline $10,00-10,99$ & 39 & 15,18 & \multirow{5}{*}{81,32} \\
\hline $11,00-11,99$ & 41 & 15,95 & \\
\hline $12,00-12,99$ & $5 \underline{2}$ & 20,23 & \\
\hline $13,00-13,99$ & $3 x$ & 11,78 & \\
\hline $1^{\prime} t, 00-11^{\prime}, 99$ & 39 & 15.18 & \\
\hline $15,00-15,99$ & 10) & 3,89 & \multirow{2}{*}{5,45} \\
\hline $16,00-16,99$ & 4 & 1,56 & \\
\hline
\end{tabular}

La valeur la plus basse était de $5,2 \mathrm{~g} / \mathrm{I}$ oo $\mathrm{ml}$ et la plus élevée de $\mathrm{I} 6,4$.

Ces limites extrêmes currespondent assez exactement aux résultats obtenus aux États-Unis par HrbBs et coll. (I96I) pour des veaux femelles. Ces chercheurs ont constaté que la teneur en hémoglobine du sang de veaux femelles nouveau-nés se situait entre 5 et I $5 \mathrm{~g}$ par Ioo $\mathrm{ml}$. Ils n'ont pas observé de corrélation entre la teneur en hémoglobine du sang des mères prélevé post-partum et celui des filles. CHARPENTIER et BONHOMme (I968) par contre, ont mis en évidence, sur I68 couples mèresveaux, une liaison significative entre l'hématocrite des veaux à 8 jours et l'hématocrite maternel $(r=0,52)$.

Nos propres observations nous apprennent encore qu'environ $8 \mathrm{I}$ p. Ioo de nos 
veaux présentaient une teneur en hémoglobine comprise entre Io et $15 \mathrm{~g} / 100 \mathrm{ml}$. Nous tenons pour important le fait que seulement environ I3 p. Ioo des veaux en cause présentaient une teneur en hémoglobine inférieure à Io g/Ioo $\mathrm{m} 1$.

HibBs et coll. (I96r) avaient, par contre, relevé dans les conditions où ils opéraient que la teneur en hémoglobine du sang était inférieure à $9 \mathrm{~g} / \mathrm{I} 00 \mathrm{ml}$ chez près de 30 p. IOo de veaux femelles. GREATOREx (I957) a constaté une teneur moyenne en hémoglobine de I 2,4 chez des veaux âgés d'une semaine et de II,5 chez des veaux âgés de deux semaines.

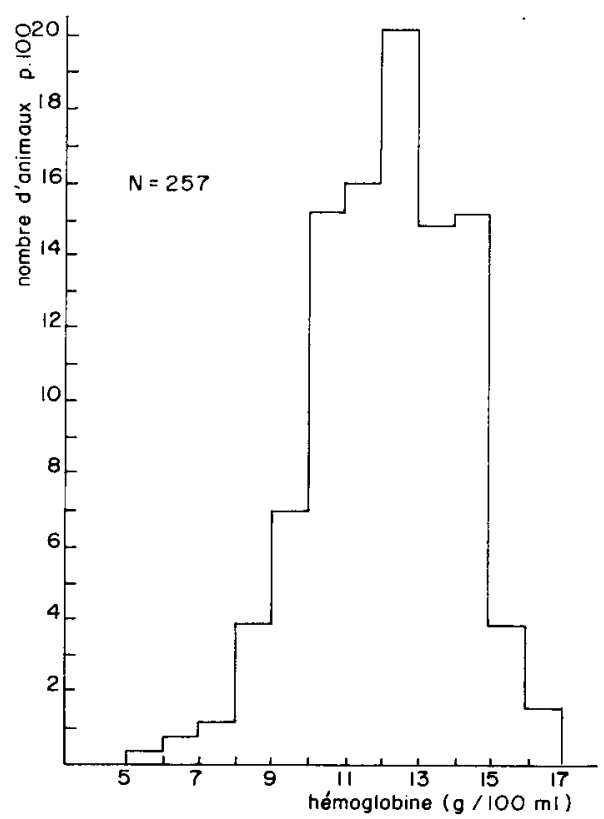

Fig. I. - Hémoglobine de veaux males de la race Pie rouge de la Flandre orientale à l'âge d'environ 10 jours

\section{Valeur de l'hématocrite.}

Én plus des données relatives à l'hémoglobine, nous disposions des valeurs de 1'hématocrite du sang de 264 veaux mâles de la race Pie rouge de la Flandre orientale, valeurs également déterminées avant le début de la période d'engraissement, c'està-dire à 1'âge d'environ Io jours. Les animaux dont nous avions déterminé les teneurs en hémoglobine du sang constituaient la très grosse majorité de ce groupe. Lues déterminations de la valeur de l'hématocrite ont d'ailleurs été effectuées sur les mêmes échantillons de sang. Quant aux autres animaux, seule la valeur de 1'hématocrite a été déterminée, de la façon décrite ci-dessus. Les résultats de ces déterminations sont reproduits dans le tableau 2 et dans la figure 2 . On doit en déduire que, de même que les taux d'hémoglobine, les valeurs de l'hématocrite du sang des veaux mâles de la race $P$ ie rouge de la Flandre orientale peuvent varier très fortement. Ia moyenne de ces 264 observations individuelles a donné une valeur de 1'hématocrite de 43,59. Les valeurs extrêmes étaient 2 I, o et 66,5 . On peut déduire en outre du tableau 2 qu'environ $78 \mathrm{p}$. Ioo des animaux examinés au début de la période d'engraissement 
présentaient une valeur de l'hématocrite comprise entre 35 et 55 et 13,28 p. Ioo une valeur hématocrite inférieure à 35 .

Greatorex (I957) fait état d'une valeur de l'hématocrite moyenne de 42,4 à l'âge d'une semaine et de 43, o à l'âge de deux semaines. Il faut conclure de ce qui précède que les caractéristiques sanguines de très jeunes veaux mâles peuvent présenter des différences considérables.

\section{TABLEAU 2}

Valours de l'hématocrite de veaux de la race Pie rouge de la Tilandre orientale $(n=264)$ âgés d'environ 10 jours

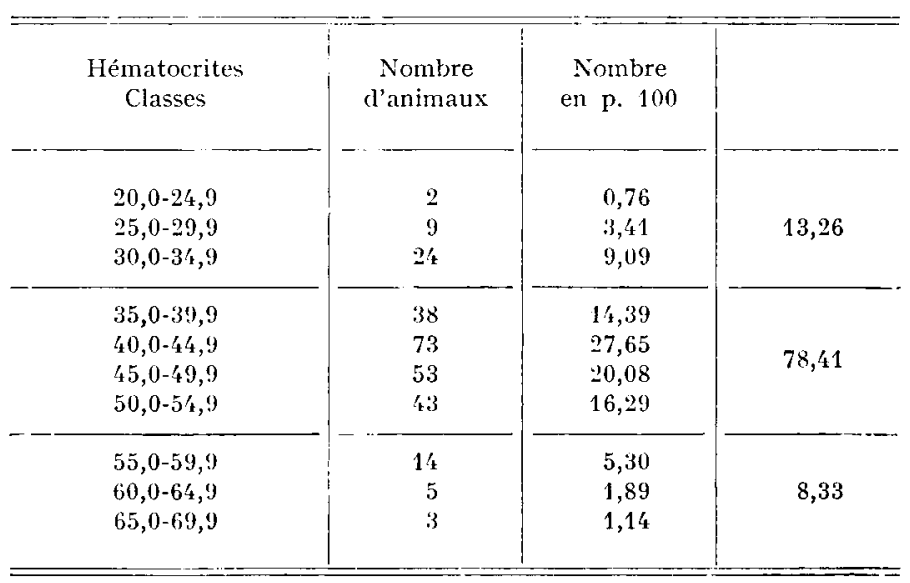

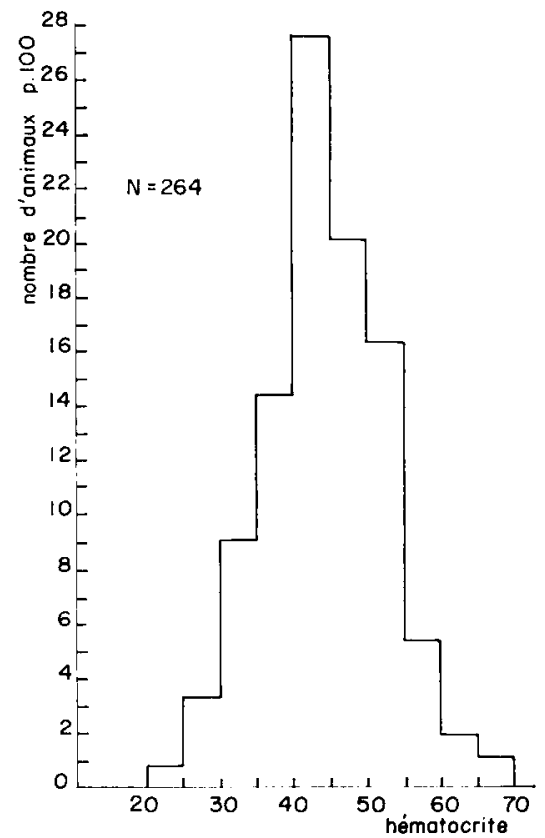

FIg, 2. - Hématocrite de veaux mâles de la race Pie rouge de la Flandre orientale à l'age d'environ 10 jours 
Il faut en conclure également qu'un certain nombre de veaux se trouve à ce moment dans un état d'anémie évident. Ceci nous a amenés à poser la question suivante : la teneur en hémoglobine et la valeur de l'hématocrite du sang de ces veaux ne baisseraient-elles pas quand on engraisse ces animaux à l'aide d'un aliment d'allaitement pauvre en fer jusqu'à un poids final d'environ $150 \mathrm{~kg}$ ?

Cette question a été le point de départ des recherches décrites ici.

\section{II. - Infuence des caractéristiques sanguines}

au début de la période d'engraissement sur les caractéristiques sanguines au moment de l'abattage (environ $160 \mathrm{~kg}$ )

et sur la couleur de la viande des veaux à l'engrais

Nous disposions de 88 veaux pour étudier ce problème. Les veaux ont été engraissés à l'aide d'aliments d'allaitement normaux à relativement faible teneur en Fe (environ 20 p.p.m. Fe). La teneur en hémoglobine du sang et la valeur de l'hématocrite ont été déterminées au début et à la fin de la période d'engraissement.

La teneur en pigment musculaire a été déterminée sur un échantillon prélevé sur le Longissimus dorsi après l'abattage. Les animaux ont été répartis en classes pour permettre d'étudier l'influence que la teneur en hémoglobine et la valeur de l'hématocrite du sang au début de la période d'engraissement exercent sur l'état d'anémie constaté lors de l'abattage et sur la couleur de la viande. La répartition en classes de ces 88 animaux a été guidée uniquement par les résultats des déterminations d'hémoglobine effectuées à l'âge d'environ Io jours (début de la période d'engraissement).

Le tableau 3 procure un aperçu de ce classement et des valeurs de 1'hématocrite moyennes correspondantes.

TABIEAU 3

Classement des veaux selon los taux d'hémoglobine initiaux

(g/100 ml)

\begin{tabular}{|c|c|c|c|}
\hline $\begin{array}{c}\text { Classes hémoglobine } \\
\text { initiale } \\
(\mathrm{g} / 100 \mathrm{ml})\end{array}$ & $\begin{array}{l}\text { Nombre } \\
\text { de veallx }\end{array}$ & $\begin{array}{l}\text { Hémoglobine } \\
\text { initiale } \\
\text { moyenne } \\
(\mathrm{g} / 100 \mathrm{ml})\end{array}$ & $\begin{array}{l}\text { Hématocrite } \\
\text { initial } \\
\text { correspondant }\end{array}$ \\
\hline$|6,00-1 / 1,0|$ & 16 & 14,75 & $5: 3,38$ \\
\hline $14,00-12,01$ & 13 & 12,57 & 17,73 \\
\hline $12,00-10,01$ & 36 & $10,8^{\prime}$ & 41,14 \\
\hline $10,00-8,01$ & 18 & $9, \geq 0$ & 36,22 \\
\hline $8,00-6,01$ & 5 & 7,31 & 30,20 \\
\hline
\end{tabular}

Le tableau 4 indique, en fonction du classement admis d'emblée, les teneurs en hémoglobine du sang à la fin de la période d'engraissement et les baisses correspondantes, exprimées en $\mathrm{g}$ par Ioo $\mathrm{ml}$ de sang. 
Il ressort du tableau 4 que la teneur en hémoglobine a baissé dans chaque classe pendant la période d'engraissement.

Cette baisse est cependant beaucoup plus marquée chez les veaux qui présentaient une forte teneur en hémoglobine au début de la période d'engraissement. La baisse est même minime chez les animaux de la classe présentant la plus faible teneur initiale (de 8 à $6,0 \mathrm{I} \mathrm{g} / 100 \mathrm{ml}$ ). En conséquence, les teneurs initiales en hémoglobine

\section{TABIEAU 4}

Baisse de la teneur en hémoglobine du sang pendant la période d'engraissement par rapport à la teneur initiale

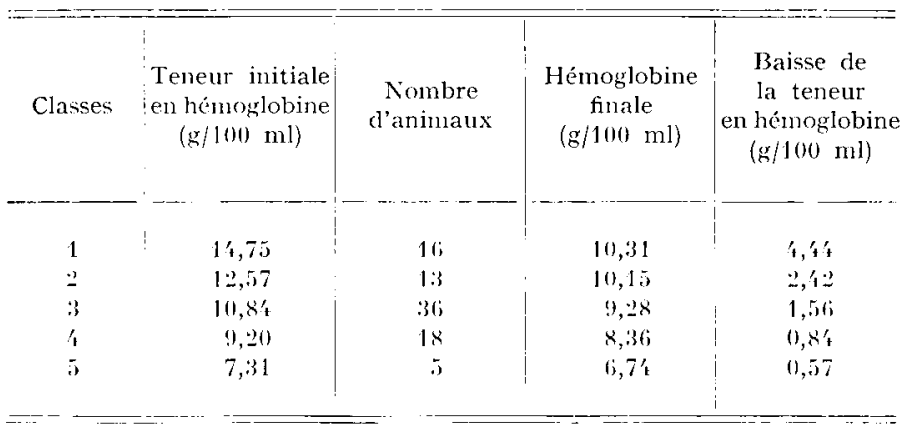

du sang ont sensiblement diminué chez la plupart des veaux à la fin de la période d'engraissement, sans toutefois s'être ézalisées.

I e tableau 5 reproduit les valeurs de l'hématocrite à la fin de la période d'en graissement ainsi que les baisses correspondantes. Il est basé, lui aussi, sur la répar tition en classes initialement admise.

TABIEAU 5

Baisse de la valour de l'hématocrite pendant la période d'engraissement par rapport à la valcur de l'hématocrite initiale'

\begin{tabular}{|c|c|c|c|c|}
\hline Classes & $\begin{array}{c}\text { Hématocrite } \\
\text { initial }\end{array}$ & $\begin{array}{l}\text { Nombre } \\
\text { d'animaux }\end{array}$ & $\begin{array}{c}\text { Hómatocrite } \\
\text { final }\end{array}$ & $\begin{array}{l}\text { Baisse de } \\
\text { l'hématocrite }\end{array}$ \\
\hline 1 & 53,38 & 16 & 35,05 & 18,33 \\
\hline$\therefore$ & $47,7: 3$ & $1: 8$ & 36,25 & 13,48 \\
\hline 3 & 41,14 & 36 & 82,16 & 8,98 \\
\hline 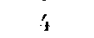 & 36,22 & 18 & 30,32 & 5,90 \\
\hline 5 & 30,20 & 5 & 23,65 & 6,55 \\
\hline
\end{tabular}

Ce tableau 5 indique que la valeur de l'hématocrite a diminué dans chaque classe pendant la période d'engraissement. Ia plus importante diminution quantitative a cependant été relevée chez les veaux qui présentaient au moment de l'achat 
la valeur de l'hématocrite la plus élevée. On constate que la baisse a été généralement faible là où les valeurs de l'hématocrite étaient moins élevées, mais que les différences entre les classes sont restées notables jusqu'à la fin.

Les tableaux 4 et 5 nous apprennent que les veaux à l'engrais recevant des aliments d'allaitement normaux deviennent anémiques ou davantage anémiques au cours de la période d'engraissement, mais que les animaux dont les caractéristiques sanguines sont défavorables dès le début se défendent apparemment mieux contre l'aggravation de l'anémie que les veaux entamant la période d'engraissement avec des teneurs en hémoglobine et des valeurs de l'hématocrite élevées.

Le risque de voir les animaux recevant des aliments d'allaitement devenir anémiques à un degré exceptionnel à la fin de la période d'engraissement semble donc moins à craindre à la lumière de ce qui précède. Un certain degré d'anémie est neéanmoins une condition indispensable à l'obtention de veaux blancs.

On peut aussi démontrer, pour les 88 animaux qui ont fourni les données des tableaux 3,4 et 5 , que les caractéristiques sanguines initiales du sang influent aussi sur la teneur en pigment et, par conséquent sur la couleur de la viande.

Le tableau 6 donne les résultats de cette démonstration.

\section{TABLEAU 6}

Infuence exercée sur la teneur en pigment musculaive par la teneur en hémoglobine et par la valeuv de l'hématocrite du sang au début de la période d'engraissement

\begin{tabular}{|c|c|c|c|c|}
\hline Classes & $\begin{array}{c}\text { Hémoglobine } \\
\text { initiale } \\
(\mathrm{g} / 100 \mathrm{ml})\end{array}$ & $\begin{array}{c}\text { Nombre } \\
\text { d'animaux }\end{array}$ & $\begin{array}{c}\text { Hématocrite } \\
\text { initial }\end{array}$ & $\begin{array}{c}\text { p.p.m. } \\
\text { d'hématine } \\
\text { dans le } \\
\text { Long. dorsi }\end{array}$ \\
\hline 1 & 14,75 & 16 & 53,38 & 46,24 \\
\hline 2 & 12,57 & 13 & 47,73 & 41,48 \\
\hline 3 & $10,8^{4}$ & 36 & 41,14 & 38,08 \\
\hline 4 & 9,20 & 18 & 36,22 & 38,76 \\
\hline 5 & 7,31 & 5 & 30,20 & 27,20 \\
\hline
\end{tabular}

On peut déduire du tableau 6 que plus les animaux sont anémiques au début de la période d'engraissement, plus on peut s'attendre à obtenir, en moyenne, une viande plus pauvre en pigment ou de couleur plus pâle.

\section{III. - Influence de la teneur en Fe de l'aliment d'allaitement} et des éléments figurés du sang au début de la période d'engraissement sur la teneur en pigment musculaire

On cherche à obtenir l'anémie désirée et la pâleur de la viande chez les veaux à l'engrais en leur donnant une alimentation pauvre en fer. L'anémie plus ou moins prononcée que les veaux présentent à la fin de la période d'engraissement est du type normochrome microcytaire (EECKHOUT et coll., I969). 
Outre l'influence exercée par l'administration de fer via l'aliment d'allaitement et par les conditions de stabulation, il faut aussi escompter une influence de la valeur initiale des caractéristiques sanguines. En d'autres termes : on peut prévoir que les taux d'hémoglobine et les valeurs de 1'hématocrite existant au début de la période d'engraissement influeront sur la teneur en pigment ou la couleur de la viande.

Pour nos recherches sur l'influence de la teneur en fer de l'aliment d'allaitement sur la teneur en pigment du Longissimus dorsi, nous disposions de Io séries de veaux, II7 animaux en tout, recevant des aliments d'allaitement, supplémentés ou non de $\mathrm{FeSO}_{4} 7 \mathrm{H}_{2} \mathrm{O}$ et dont la teneur en $\mathrm{Fe}$ était connue (tabl. 7 ).

TABLEAU 7

Teneurs en fer des aliments d'allaitement utilisés

\begin{tabular}{|c|c|c|c|}
\hline $\begin{array}{l}\text { p.p.m. de fer } \\
\text { dans l'aliment } \\
\text { d'allaitement }\end{array}$ & $\begin{array}{c}\text { Fe supplémentaire } \\
\text { sous forme de } \\
\mathrm{FeSO}_{4} \cdot 7 \mathrm{H}_{2} \mathrm{O} \\
\text { (p.p.m.) }\end{array}$ & $\begin{array}{c}\text { Fe total } \\
\text { dans l'aliment } \\
\text { d'allaitement } \\
\text { (p.p.m.) }\end{array}$ & $\begin{array}{c}\text { Nombre } \\
\text { d'animaux }\end{array}$ \\
\hline 15 & 0 & 15 & 27 \\
\hline 21 & 0 & 21 & 7 \\
\hline 22 & 0 & 22 & 15 \\
\hline 23 & () & 23 & 7 \\
\hline 25 & () & 25 & 11 \\
\hline$\because 1$ & 15 & 36 & 7 \\
\hline 21 & 30 & 51 & 6 \\
\hline 21 & 45 & 66 & 7 \\
\hline 15 & (6) & 75 & 14 \\
\hline 22 & 60 & 82 & 16 \\
\hline
\end{tabular}

Si nous partons de l'hypothèse discutable que le Fe présent d'emblée dans l'aliment d'allaitement est assimilable de la même façon que le $\mathrm{Fe}$ du sulfate ferreux de supplément, on peut essayer de rechercher à l'aide du matériel expérimental disponible quelle influence les diverses teneurs en Fe exercent sur la teneur en pigment et donc sur la couleur de la viande. Un calcul de régression curvilinéaire entre la teneur en fer de l'aliment d'allaitement (p.p.m.) du Longissimus dorsi a abouti à l'équation suivante :

$$
\begin{aligned}
& \mathrm{Y}=47, \mathrm{I} 468-0,4990 \mathrm{X}+\text { o,009 o8 } \mathrm{X}^{2} \\
& \mathrm{Y}=\text { hématine du Longissimus dorsi (p.p.m.) } \\
& \mathrm{X}=\text { fer de 1'aliment d'allaitement (p.p.m.) }
\end{aligned}
$$

On trouvera à la figure 3 une représentation graphique de l'équation de régression obtenue par calcul.

On serait tenté d'admettre, d'après ces données, qu'une teneur en Fe de l'aliment d'allaitement variant entre 20 et 35 p.p.m. n'influe que peu sur la teneur en pigment. Nous voulons nous-mêmes mettre ceci en doute (EECKHOUT et coll., I969). Une explication possible peut résider dans le fait qu'il n'a pas été tenu compte de l'état des veaux (teneur en hémoglobine et valeur de l'hématocrite de leur sang) au 
début des essais. La grande importance que ces facteurs ont pour la couleur de la viande sera mise en lumière par ce qui suit.

Si nous nous basons sur la teneur moyenne en hémoglobine au début des essais pour scinder nos données obtenues sur II 7 veaux, nous pouvons répartir ces animaux en deux groupes : un groupe où la teneur initiale en hémoglobine était supérieure à la moyenne et un groupe où cette teneur initiale était inférieure à la moyenne. Cette

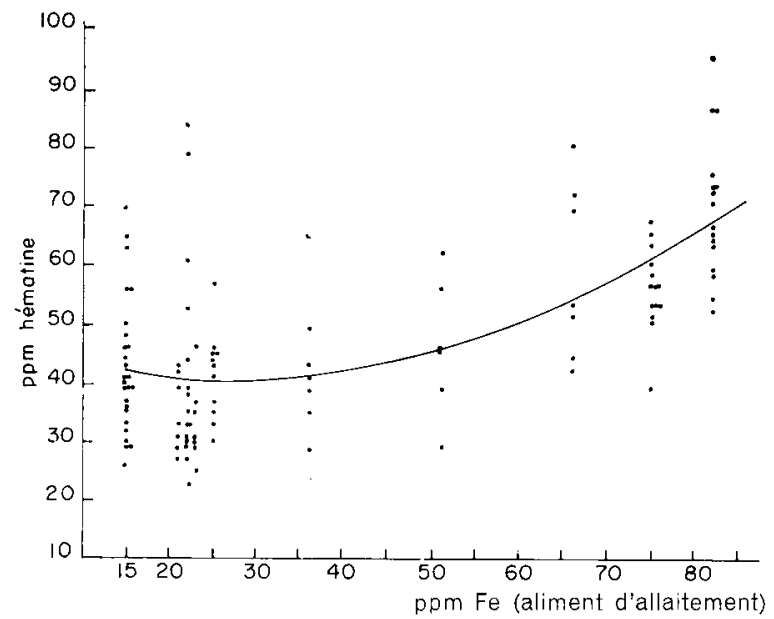

FIG. 3. - Infuence de la teneur en fer de l'aliment d'allaitement sur la teneur en hématine du muscle longissimus dorsi

TABLEAU 8

Influence exercée sur la teneur en pigment du Longissimus dorsi par la tencur en hémoglobine et la valeur de l'hématocrite du début de la période d'engraissement a's veaux

\begin{tabular}{|c|c|c|c|c|c|c|c|c|}
\hline \multirow{2}{*}{$\begin{array}{l}\text { Teneurs en Fe } \\
\text { de l'aliment } \\
\text { d'allaitement } \\
\text { (p.p.m.) }\end{array}$} & \multicolumn{2}{|c|}{$\begin{array}{c}\text { Hémoglobine } \\
\text { initiale } \\
>\text { 12,́1́ g/100 ml }\end{array}$} & \multicolumn{2}{|c|}{$\begin{array}{c}\text { Hémogrobine } \\
\text { initiale } \\
<12, \text { 't' } / 100 \mathrm{ml}\end{array}$} & \multicolumn{2}{|c|}{$\begin{array}{l}\text { Hématocrite } \\
\text { initial } \\
>\quad 41,22\end{array}$} & \multicolumn{2}{|c|}{$\begin{array}{l}\text { Hématocrite } \\
\text { initial } \\
<44,22\end{array}$} \\
\hline & $\begin{array}{c}\text { Hématine } \\
\text { viande } \\
\text { (p.p.n.) }\end{array}$ & $\begin{array}{l}\text { Nombre } \\
\text { d'ani- } \\
\text { maux }\end{array}$ & $\begin{array}{l}\text { Hómatine } \\
\text { viande } \\
\text { (p.p.n.) }\end{array}$ & $\begin{array}{l}\text { Nombre } \\
\text { d'ani- } \\
\text { maux }\end{array}$ & $\begin{array}{l}\text { Hématine } \\
\text { viande } \\
\text { (p.p.nn.) }\end{array}$ & $\begin{array}{l}\text { Nombre } \\
\text { d'atij- } \\
\text { maux }\end{array}$ & $\begin{array}{l}\text { Hématine } \\
\text { viande } \\
\text { (p.p.m.) }\end{array}$ & $\begin{array}{l}\text { Nombre } \\
\text { d'ani- } \\
\text { maux }\end{array}$ \\
\hline 15 & 47 & 13 & 39 & 1' & 45 & 16 & 10 & 11 \\
\hline-1 & 38 & 3 & 3 & 't & 38 & 't & 30 & 3 \\
\hline 22 & 52 & $x$ & 32 & 7 & 55 & 7 & 32 & 8 \\
\hline 23 & 35 & 1 & 33 & 6 & 40 & 2 & 30 & 5 \\
\hline 25 & $4: 3,5$ & 8 & 36 & 3 & 42 & 9 & 38 & 2 \\
\hline 36 & 47 & ' & 38 & 3 & 51 & 2 & 39 & 5 \\
\hline 51 & 46 & 6 & - & - & 50 & 4 & 38 & 2 \\
\hline 66 & 67,5 & '́ & 46 & 3 & 74 & $\ddot{\prime}$ & 52 & 5 \\
\hline 75 & 54 & 5 & 57 & 9 & 58 & 6 & $5^{\prime} t$ & 8 \\
\hline 82 & 68 & 10 & 72 & 6 & 65 & 6) & 72 & 10 \\
\hline & & 62 & & 55 & & 58 & & 59 \\
\hline
\end{tabular}


teneur initiale moyenne était de $\mathrm{I} 2,44 \mathrm{~g} /$ I $00 \mathrm{ml}$ pour l'ensemble des I I7 veaux; elle était dépassée chez 62 veaux tandis que 55 veaux présentaient un taux initial moindre.

Une répartition analogue a été effectuée selon les valeurs de l'hématocrite. La valeur de l'hématocrite initiale moyenne à l'âge d'environ Io jours, soit 44,22 pour les II 7 veaux, était dépassée chez $5^{8}$ animaux et n'était pas atteinte chez 59 . Le tableau 8 expose l'influence de la teneur initiale en hémoglobine du sang et de la valeur initiale de l'hématocrite sur la teneur en pigment du Longissimus dorsi, ceci pour les différentes teneurs en $\mathrm{Fe}$ de l'aliment d'allaitement.

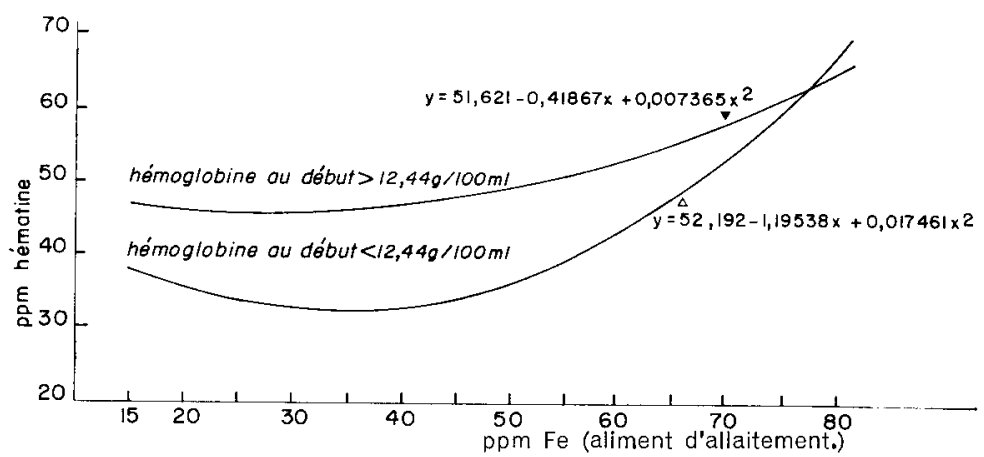

FIc. 4. - Teneur en pigment du muscle chez les veaux présentant un taux initial d'hémoglobine supérieur ou inférieur à la moyenne $(12,44 / 100 \mathrm{ml})$, en fonction de la teneur en fer de l'aliment d'allaitement

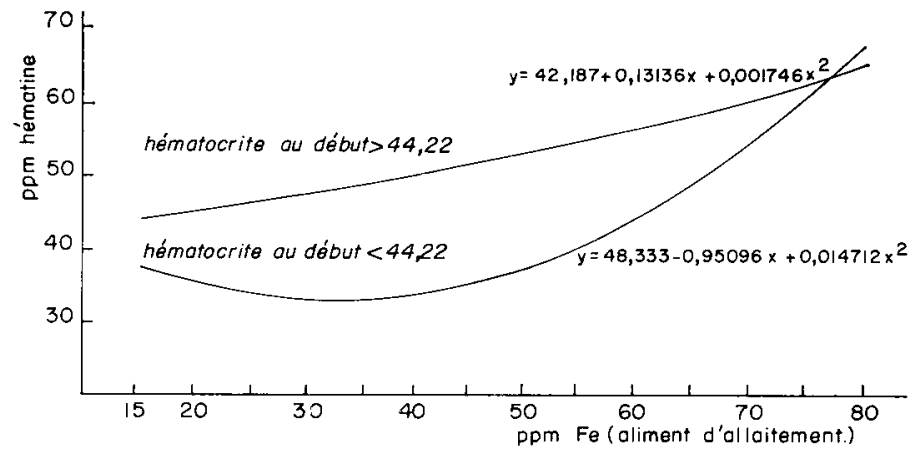

Fig. 5. - Teneur en pigment du muscle chez les veaux présentant une valeur initiale de l'hématocrite supérieure ou inférieure à la moyenne (44,22) en fonction de la teneur en fer de l'aliment d'allaitement

Pour presque chaque taux de $\mathrm{Fe}$, la teneur en pigment musculaire était plus élevée quand la teneur initiale en hémoglobine et la valeur initiale de l'hématocrite dépassaient la moyenne, que dans le cas contraire. Des exceptions n'ont été relevées que pour l'aliment d'allaitement présentant les plus fortes teneurs en fer contrôlées (de 73 à 82 p.p.m.). Il est possible qu'à de telles doses de fer, l'influence exercée par les éléments figurés du sang au début de la période d'engraissement soit négligeable.

Un calcul de régression curvilinéaire effectué avec les 4 séries de données (hémoglobine initiale supérieure et inférieure à la moyenne et hématocrite initial supérieur et inférieur à la moyenne) a abouti à 4 équations de régression représentées sous forme graphique dans les figures 4 et 5 . Ces figures montrent encore plus clairement 
l'influence exercée sur la teneur finale en pigment de la viande par la teneur en hémoglobine et la valeur de l'hématocrite du sang au début de la période d'engraissement. Dans les deux systèmes de répartition, la courbe relative aux animaux plus anémiques se situe nettement plus bas que celle calculée pour les animaux dont les taux d'hémoglobine et les valeurs de l'hématocrite dépassaient les moyennes relevées pour les II7 animaux en cause. Ce phénomène ne s'observe plus et les courbes se coupent lorsque les teneurs en fer de l'aliment d'allaitement sont très élevées.

\section{CONCLUSIONS}

Il ressort des données ci-dessus que la teneur en hémoglobine et la valeur de l'hématocrite du sang varient considérablement chez des veaux de la race de la Flandre orientale âgés d'environ Io jours. On relève à cet âge des cas d'anémie évidente. Le taux d'hémoglobine et la valeur de l'hématocrite du sang baissent sous l'influence d'une alimentation à aliment d'allaitement pauvre en fer. Cette baisse est toutefois la plus prononcée chez les animaux qui présentaient les taux d'hémoglobine et les valeurs de 1'hématocrite les plus élevés au début de la période d'engraissement.

La diminution du taux d'hémoglobine et de la valeur de l'hématocrite est très faible chez les animaux déjà anémiques au début de la période d'engraissement. On ne risque donc guère de voir ces animaux déjà anémiques au départ arriver à un état d'anémie maximale (que les physiologistes qualifieraient d'à peine viable), si on les nourrit d'un aliment d'allaitement contenant environ 20 p.p.m. cie fer.

Cette anémie peut néanmoins avoir de l'importance parce qu'elle peut signifier une prédisposition à d'autres maladies (danger d'infection) (WEISs, Ig67) et favorise aussi, quand les conditions climatologiques sont défavorables, 1'hyperthermie chez des animaux plus lourds (HERSCHEL, I965).

Comparés aux veaux nourris au lait entier, les veaux à qui l'on donne des aliments d'allaitement reçoivent des quantités de fer considérablement plus élevées. Ces quantités sont apparemment suffisantes pour limiter l'ampleur de la diminution de la teneur en hémoglobine et de la valeur de l'hématocrite du sang des veaux déjà anémiques au début de la période d'engraissement. Ceci concorde avec les observations d'AMMERMAN et al. (I967) qui ont montré que les veaux anémiques utilisent bien mieux que les animaux moins anémiques le fer ađministré par voie orale.

La teneur en hémoglobine et la valeur de l'hématocrite réelles du sang au début de la période d'engraissement exercent d'ailleurs une influence indiscutable sur la teneur en pigment de la viande au terme de la période d'engraissement.

Aux diverses teneurs en fer de l'aliment d'allaitement utilisées pour les essais, la teneur en pigment de la viande était, en moyenne, nettement plus élevée chez les animaux dont le taux d'hémoglobine et la valeur de 1'hématocrite dépassaient les moyennes du groupe d'animaux (respectivement $\mathrm{I} 2,44 \mathrm{~g} / \mathrm{I}$ oo $\mathrm{ml}$ et 44,22 ). Ce n'est que pour de très fortes teneurs en fer de l'aliment d'allaitement que 1'on n'a plus décelé d'effet exercé sur la teneur en pigment de la viande par les éléments figurés initiaux du sang. Tout ceci permet de supposer que le manque d'uniformité de la couleur de la viande d'un groupe de veaux engraissés simultanément à l'aide d'un 
même aliment d'allaitement dissous dans une même eau, est imputable, au moins en partie, à la différence entre les éléments figurés du sang de ces veaux au moment où l'engraissement a commencé.

Si les courbes reproduites ici ne mettent pas clairement en évidence l'influence de la teneur en Fe de l'aliment d'allaitement sur la teneur en pigment musculaire du moins pas en ce qui concerne la gamme de teneurs en Fe situées entre I5 et 35 p.p.m. - , nous devons tout de même mettre en garde contre des conclusions prématurées. Il faut, en effet, considérer que les veaux qui ont reçu ces aliments d'allaitement définis globalement par la présence d'une dose de fer déterminée, n'avaient pas toujours des caractéristiques sanguines initiales comparables. Or nous avons bien relevé, dans des conditions où il était effectivement tent compte de ces facteurs (EECKHOUT et coll., I969), une influence exercée sur la couleur de la viande par l'addition de 15 p.p.m. de fer à un aliment d'allaitement normal, qui en contenait déjà environ 20 p.p.m. Nous croyons donc pouvoir affirmer que certains veaux (anémiques au départ) supportent plus aisément une teneur en fer légèrement plus forte de l'aliment d'allaitement que les veaux à taux d'hémoglobine et valeurs de l'hématocrite élevés lors de la mise à l'engrais.

$\mathrm{Si}$, par contre, on veut arriver à donner, en plus d'une eau très pauvre en fer (o,I p.p.m.), un aliment d'allaitement dont la teneur en fer est nettement inférieure à 20 p.p.m. (DAMMERs, I965), par exemple Io p.p.m., on obtiendra peut-être encore moins de veaux dont la viande présente une couleur décevante, mais on augmentera sans doute aussi les risques de troubles imputables à un état d'anémie trop avancée.

(Traduit en néerlandais.)

Reçu pour publication en juin 1969.

\section{SUMMARY}

\section{ANEMIA AND MEAT COLOR IN VEAL CALVES OF THE PIE ROUGE DE FLANDRE ORIENTALE BREED}

The hemoglobin content of the blood of 257 male calves, and the hematocrite value of 264 male calves were determined at Io days of age. The results are shown in tables $\mathrm{I}$ and 2 , and in figure $\mathrm{I}$ and 2. The extreme values for hemoglobin content were $5.2 \mathrm{~g} / \mathrm{I}$ oo $\mathrm{ml}$ and $\mathrm{I} 6.4 \mathrm{~g} / \mathrm{I} 00 \mathrm{ml}$. The hematocrite values recorded were determ!ned after 30 minutes of centrifugation at 3500 r.p.m. in Wintrobe tubes.

They varied between $2 \mathrm{I}$ and 66.5. Tables $\mathrm{I}$ and 2 show that calves may already be noticeable anemic at 10 days. 88 calves were fattened in wooden boxes and fed a milk replacer containing $2 \mathrm{I}$ p.p.m. of iron in the dry matter and dissolved in water containing 0.65 p.p.m. Fe. Those animals which were originally anemic did not become more anemic.

This is shown by a small drop in hemoglobin content and hematocrite value. However, this drop is more pronounced in animals having a high hemoglobin content and hematocrite value at the beginning of fattening (see tables 4 and 5 ). It follows that the original differences in hemoglobin content between animals submitted to the experiment are markedly reduced during the fattening period.

The influence of hemoglobin content and hematocrite value on the Longissimus dorsi pigment content (HORNSEY, I956) at the beginning of fattening was studied using milk replacers containing very different amounts of iron. In most of the doses tried, Longissimus dorsi pigment content was higher in animals having a hemoglobin and hematocrite higher than average at ro days ( $12.44 \mathrm{~g}$ of hemoglobin/roo $\mathrm{ml}$, hematocrite $44.22 ; \mathrm{N}=\mathrm{I} 77$ ). The results are given in table 7 and figures 4 and 5 .

No influence initial of hemoglobin content and initial hematocrite value on Longissimus dorsi pigment was observed when the iron content of the milk replacer ranged between 75 and 85 p.p.m. in the dry matter. 


\section{RÉFÉRENCES BIBLIOGRAPHIQUES}

Ammermax C. 13. ct al., I967. Utilization of inorganic iron by ruminants as influenced by form of iron and iron status of the animal. J. Anim. Sci., 26, 404 .

Bandemer Selma L., Scinaible P. J., ig44. Determination of iron. A study of the $o$-phenanthroline method. Ind. Fng. Chem. (Anal. Ed), 16, 3I7.

Blaxter I. L., Sharman G. A. M., Maclonald A. M., I957. Iron-deficiency anemia in calves. Br. J. Nutr., 11, 234.

Charpentiek J., ig66, Pigmentation musculaire du veau de boucherie. II. Influence d'une supplémentation alimentaire en fer sur la teneur en fer héminique de quelques muscles. Ann. Zootech., 15, 36r.

Charpentier J., Bonhomme D., I968. liacteurs de variation de l'hématocrite des bovins. I. Hématocrite des veaux à 8 jours, Ann. Zootech., 17, $32 \mathrm{r}$.

DAmmers J., 1965. Voeding van mestkalveren. Veetelt-en Zuivelberichten, 8, 262.

Eeckhout W., Castiels M., Buysse I., ig69. Influence de la teneur en fer des aliments d'allaitement pour veaux à l'engrais sur les éléments figurés du sang, la couleur de la viande et les résultats d'engraissement. Ann. Zootech., 18, (3), 249-26r.

Gorther E., De GraAfF W. C., I965. Klinische diagnostick. Deel, I, p. 30o. Standaard boekhandel, Antwerpen.

GrLatorex J. C., I957. Observations on the hematology of calves and various breeds of adult dairy cattle. Br. vet. J., 113, 65.

Herschel A., 1965. Verzorging en zickten van mestkalveren. Veeteelt-en Zuivelberichten, 8, I8I.

Hibbs J. W., Conrad H. R., Gale C., 196r. liurther studies on anemia in newborn dairy calves. J. Dairy Sci, 44, II 84 .

Hornsey H., r956. The colour of cooked cured pork. J. Sci. Fd Agric., 7, 534.

Pyck J., Hoste J., Gillis J., r958. Onderzoek met behulp van radio-actieve tracers omtrent het verlies van sporenelementen bij de destructie van biologisch materiaal. Meded. K. zlaam. Acad., $29,8$.

Ror J. H. B. and $a l$, rg64. The nutrition of the veal calf. The effect of anemia and of iron and chlortetracycline supplementation on the performance of calves given large quantities of whole milk. $\mathrm{Br}$. J. Nutr., 18, 467 .

Schmitten G., Noack D., I967. Über der verlauf des Hämatokrit-Gehaltes bei Mastkälbern und seine Beeinflussung durch Fütterung und Haltung. Dt. Tierärztl. Wschr., 74, I 12.

VAN de Voorde G., I968. (Communication personnelle).

Verbeke R., Martin J, I967. Contribution à l'étude de la couleur de la viande de veaux blancs. Commu. nication $\mathrm{n}^{\mathbf{0}} 8$. Centre d'études bovines. Coupure, 235, Gent.

Weiss E., r967. Nicht-infektiöze Kalberkrankheiten. Mitt. f. Tierhaltung. Heft 108, p. 2 I. 